\title{
Perioperative considerations of an extended forequarter amputation for recurrent high-grade radiation-induced sarcoma: A case report
}

\author{
Alexander Stoker ${ }^{*}$, Jeff Mueller ${ }^{1}$, Christopher Thunberg ${ }^{1}$, Krista Goulding ${ }^{2}$, Staci Beamer ${ }^{3}$, Michael Hinni $^{4}$, Alanna Rebecca ${ }^{5}$, Christopher \\ Beauchamp $^{2}$ and Andrew Gorlin ${ }^{1}$ \\ ${ }^{1}$ Department of Anesthesiology and Perioperative Medicine, Mayo Clinic Arizona, Phoenix, USA \\ ${ }^{2}$ Department of Orthopedic Surgery, Mayo Clinic Arizona, Phoenix, USA \\ ${ }^{3}$ Department of Cardiothoracic Surgery, Mayo Clinic Arizona, Phoenix, USA \\ ${ }^{4}$ Department of Otorinolaryngology, Mayo Clinic Arizona, Phoenix, USA \\ ${ }^{5}$ Department of Plastic and Reconstructive surgery, Mayo Clinic Arizona, Phoenix, USA
}

\begin{abstract}
Forequarter amputation is a radical surgical procedure involving removal of the entire upper extremity and shoulder girdle and is most commonly performed in the management of aggressive upper extremity malignancies. A number of perioperative challenges can arise during extended resections including postoperative respiratory compromise due to altered chest wall mechanics, potential need for lung isolation, selecting appropriate sites for vascular access, complex fluid and hemodynamic resuscitation, optimizing conditions for free flap viability, and managing pain and the psychological impact of such a significant operation. Here we present a case of an extended forequarter amputation with a multidisciplinary team for the management of a recurrent high-grade radiation-induced sarcoma.
\end{abstract}

\section{Introduction}

Forequarter (interscapulothoracic) amputation is a radical surgical procedure involving removal of the entire upper extremity and shoulder girdle. It was initially used in the early $19^{\text {th }}$ century to manage severe traumatic injuries but now is most commonly indicated in the management of malignant tumors of the arm, axilla, shoulder and scapula [1]. Occasionally, tumors will also invade the chest wall requiring an extended en-bloc resection of the extremity, chest wall and rarely, the lung to achieve negative margins.

Radiation-induced sarcoma is an aggressive malignancy originating in irradiated bone or soft tissues, and the majority of tumors are high grade [2]. Patients with radiation-induced sarcomas have a 5-year survival rate of $17 \%$ to $58 \%$ with surgical resection being the primary means of treatment [3].

A number of perioperative challenges can arise during extended forequarter amputations including postoperative respiratory compromise due to altered chest wall mechanics, potential need for lung isolation, selecting appropriate sites for vascular access, optimizing conditions to ensure flap viability, and managing pain and the psychological impact of such a significant operation.

All information was de-identified in this case report.

\section{Case report}

A 66-year-old woman (59 kg and BMI of 24) presented to our institution for evaluation of a recurrent radiation-induced $9 \times 9 \times 12$ $\mathrm{cm}$ high-grade spindle cell sarcoma involving the right axilla, scapula, glenohumeral joint, base of neck, chest wall, and right upper lung pleura with encasement of the brachial plexus and axillary vessels.
Her other past medical history was significant for a remote history of right arm deep vein thrombosis for which she was anticoagulated with warfarin, hypertension, history of doxorubicin-induced systolic heart failure (transthoracic echo showing left ventricular ejection fraction of 40-45\%), chronic right arm pain due to tumor involvement of the brachial plexus (taking MS contin $30 \mathrm{mg}$ TID as well as morphine immediate release for a daily morphine equivalent of $180 \mathrm{mg}$ ), chronic iron deficiency anemia with a preoperative hemoglobin of 10.5, and generalized anxiety disorder (on lorazepam $0.5 \mathrm{mg}$ TID PRN).

She was scheduled for an extended forequarter amputation involving a multidisciplinary surgical approach involving orthopedic surgery, plastic surgery, cardiothoracic surgery, neurosurgery and otorhinolaryngology. General anesthesia was induced with $100 \mathrm{mcg}$ fentanyl, $100 \mathrm{mg}$ lidocaine, $100 \mathrm{mg}$ propofol, and $50 \mathrm{mg}$ rocuronium intravenously. A left-sided 37 French double lumen endotracheal tube was placed and appropriate position was confirmed with fiberoptic bronchoscope. The bronchial cuff remained deflated until lung isolation was required. Left radial arterial, left antecubital 16 gauge peripheral IV and left femoral two-lumen central line catheters were placed. Core temperature was monitored and maintained between $35.1^{\circ} \mathrm{C}$ (first recorded temperature) and $37.1^{\circ} \mathrm{C}$ with a gradual increasing trend during the procedure. The patient was given $2 \mathrm{~g}$ of cefazolin and $2 \mathrm{~g}$ of tranexamic acid intravenously.

${ }^{*}$ Correspondence to: Alexander Stoker, Department of Anesthesiology and Perioperative Medicine, Mayo Clinic Arizona, Phoenix, USA, E-mail: Stoker.Alexander@mayo.edu

Received: August 13, 2020; Accepted: August 24, 2020; Published: August 27, 2020 
The patient was positioned in left lateral decubitus position and the right arm, chest wall and neck were prepped and draped. The plastic surgery team began by preparing the forearm for a free fillet flap, but delaying the anastomosis until the extremity was removed. The surgical teams then performed a right forequarter amputation with neck dissection and lymphadenectomy, costotransversectomy from $\mathrm{C} 7$ to $\mathrm{T} 2$, and chest wall resection. The brachial plexus, external jugular vessels and internal jugular vein were divided. Left lung isolation was required during the chest wall resection involving the first through fifth ribs, sternoclavicular joint, a portion of the manubrium, right upper lobectomy, and total lung pulmonary decortication. The right upper extremity was then removed from the body, leaving a $17 \times 25$ centimeter defect. The plastic surgery team then removed the free fillet flap from the extremity on a sterile back table, and inset the free forearm fillet flap, anastomosing the brachial artery and vein of the free fillet flap to the lingual artery and anterior cervical vein, respectively. Then 2000 units of heparin were administered intravenously. Reconstruction of the chest wall was performed with a $15 \times 18 \mathrm{~cm}$ Goretex mesh, and the flap was inset and incisions reapproximated. The surgical time of the procedure was 10 hours and 21 minutes.

Estimated blood loss was $1200 \mathrm{ml}$. Urine output was $2850 \mathrm{ml}$. A total of two units of packed red blood cells were transfused. The hemoglobin ranged from 6.7 to $12.2 \mathrm{~g} / \mathrm{dL}$ during the case. The patient received $1.5 \mathrm{~L}$ of albumin and $4.8 \mathrm{~L}$ of plasmalyte during the procedure. Intermittent hypotension was treated with low-dose phenylephrine and ephedrine. For analgesia the patient was given a total of $4 \mathrm{mg}$ of hydromorphone and $50 \mathrm{mg}$ of ketamine intravenously throughout the case as well as a dexmedetomidine infusion at $0.4 \mathrm{mcg} / \mathrm{kg} / \mathrm{hr}$ during the case.

At the conclusion of surgery a Cook airway exchange catheter was used to place a single lumen endotracheal tube and the patient was taken to the intensive care unit intubated. Three hours later the patient developed a significant intra flap hematoma necessitating massive transfusion and a return to the operating room where multiple areas of bleeding were identified and controlled. Subsequently, the patient was noted to have venous congestion in the flap and a clot was removed from the venous anastomosis site. Her free flap ultimately failed and was removed despite resuscitation, optimization of perfusion using leach therapy and mixed venous oxygen monitoring of the flap. On post op day 4 the patient was taken for a chest wall closure using a rectus abdominal myocutanous flap and open tracheostomy. She remained in the intensive care unit receiving ventilator support for 32 days and then was discharged home on post op day 64 . On 3 month follow up the patient had no evidence of recurrence and a well healed flap.

\section{Discussion}

Sarcomas arising from the chest wall and axilla can require complex procedures to achieve negative margins when surgery has a curative intent. Extended forequarter amputations where the neck, spine and/ or chest wall are involved are particularly challenging and require a multidisciplinary approach including the anesthesiologist, who plays an important role in the care of these patients. A number of perioperative challenges arise during forequarter amputations.

\section{Pulmonary considerations}

Muscles contributing to respiration that may be disrupted by an extended forequarter amputation include the intercostal, sternocleidomastoid, pectoralis, seratus anterior, scalene, and upper trapezius muscles [4]. Loss of function in these muscles may reduce pulmonary function and may possibly contribute to postoperative respiratory complications. In a cross sectional cohort study of patients with spinal cord injuries and loss of accessory muscles of respiration the mean $\mathrm{FEV} 1$ was $55 \%$ predicted in $\mathrm{C} 4$ to $\mathrm{C} 5$ injuries and $78 \%$ predicted in $\mathrm{T} 1$ to $\mathrm{T} 6$ spinal cord injuries, respectively [5]. Resection of ribs, chest wall and accessory muscles of respiration alter pulmonary mechanics and increase the likelihood of needing ventilator support in the postoperative period. Additionally, an extended resection may compromise phrenic nerve and diaphragmatic function, significantly altering respiratory function. Patients should be counseled about the possibility of prolonged ventilation and even a tracheostomy.

Depending on the depth of resection and proximity to the pleura, lung isolation may be needed. Positioning of the patient may limit access to the patient's head and make it more challenging to exchange a double lumen ETT although there may be increased risk for complications associated with leaving a double lumen ETT in place for an extensive surgery.

\section{Hemodynamic considerations}

Fluid shifts, blood loss and hemodynamic instability can be significant and in all cases arterial access and large bore venous access are mandatory. The anesthesiologist may also consider placement of a central venous catheter to assist with optimization of monitoring and resuscitation. The optimal location for a central line should be determined on a case-by-case basis. Clearly, ipsilateral jugular or subclavian access is not an option, but in certain cases contralateral jugular or subclavian access may be feasible. In some cases the femoral vein may be the best location for central access though this has to be weighed against the risk of infection. Hypotension is common during this operation and, in addition to fluid resuscitation, judicious use of vasopressors is often necessary.

\section{Free flap considerations}

A number of strategies have been proposed to increase the chance of flap viability, including optimizing both macrocirculation and microcirculation to ensure adequate flap perfusion, temperature management, optimizing hemoglobin and oxygen carrying capacity.

In a recent retrospective review of 104 patients undergoing free flap surgery for cancer of the head and neck a goal-directed fluid management strategy using stroke volume variation led to reduced intra-operative fluid administration and shorter hospital length of stay [6]. Another retrospective review of 168 patients undergoing free flap surgeries found that the lowest perioperative hemoglobin was a significant predictor of flap failure and suggested that an appropriate lower limit of transfusion commencement is $8.75 \mathrm{~g} /$ $\mathrm{dL}$ [7]. Since bleeding and thrombosis are major reasons for flap compromise and alterations in the coagulation cascade are common both intraoperatively and postoperatively, thromboelastography has been advocated as a useful tool in monitoring for coagulopathy [8]. Whether vasopressor use negatively effects free flap microcirculation remains controversial, however a recent retrospective propensity-score matching analysis of 2376 patients undergoing free flap surgery did not see any association with vasopressor use and pedicle compromise or flap failure rates however they did see less risk of venous congestion in the vasopressor group [9]. If there are clinical concerns about the effect of alpha-agonists such as phenylephrine on flap microcirculation, the anesthesiologist may administer an inotrope such as dopamine or dobutamine if the patient needs blood pressure support. 


\section{Pain management}

Patients undergoing forequarter amputations may have significant perioperative pain as well as a high incidence of phantom limb pain. In a review of 30 patients undergoing forequarter amputations in one center found that all patients had evidence of phantom limb pain [10]. A case report of 4 pediatric patients who underwent forequarter amputations described all patients having phantom limb pain, however it did not persist past 4 weeks in any patient [11].

Case reports have described using brachial plexus catheters, including interscalene catheters infusing local anesthetics for up to 14 days after forequarter amputations [10,12]. A recent case report describes successfully using a cervical erector spinae plane block catheter during a forequarter amputation [13].

In addition to regional anesthesia a multimodal analgesic approach may include the use of agents with NMDA receptor antagonism such as ketamine, methadone or magnesium, alpha-2 agonists such as dexmedetomidine, lidocaine infusions, anticonvulsants such as gabapentin, and NSAIDs.

\section{Psychological and ethical considerations}

Patients may have significant psychological stress knowing they are undergoing a radical and life-altering operation with a risk of morbidity and mortality. Operative mortality has been described as between 0 and $10 \%[14,15]$. In a retrospective review of 40 patients undergoing forequarter or hindquarter amputations the operative mortality was $2.5 \%$. This study described the most common complications being wound infection and flap necrosis with $35 \%$ of patients developing a wound complication [14].

Amputations have a significant psychological impact. The prevalence of psychiatric disorders among amputees has been described as between 32 and $64 \%$ with depression ranging from $10.4 \%$ to $63 \%$ and posttraumatic stress disorder ranging from 3.3 to $56.3 \%$ [16]. Extensive discussions with the patient by both the surgical teams and the anesthesiologist may ease perioperative anxiety and help the patient understand the goals of the operation and recovery period including managing pain, functional limitations and underlying medical conditions.

\section{Conclusion}

An extended forequarter amputation involves a multidisciplinary team and the anesthesiologist plays an important role in navigating a number of perioperative challenges including challenging airway management, intraoperative lung isolation, complex hemodynamic and fluid resuscitation, challenging pain management, anticipating postoperative respiratory compromise, and employing appropriate temperature, fluid and coagulation management to optimize conditions for free flap viability.

\section{References}

1. Dimas V, Kargel J, Bauer J, Chang P (2007) Forequarter amputation for malignan tumours of the upper extremity: case report, techniques and indications. Can J Plast Surg 15: 83-85.

2. Patel S (2000) Radiation-induced sarcoma. Curr Treat Options Oncol 1: 258-261.

3. Bjerkehagen B, Småstuen M, Hall K, Skjeldal S, Smeland S, et al. (2012) Why do patients with radiation-induced sarcomas have a poor sarcoma-related survival?. $\mathrm{Br} \mathrm{J}$ Cancer 106: 297-306.

4. de Paleville DGT, McKay WB, Folz RJ, Ovechkin AV (2011) Respiratory motor control disrupted by spinal cord injury: mechanisms, evaluation, and restoration. Transl Stroke Res 2: 463-473.

5. Jain NB, Brown R, Tun CG, Gagnon D, Garshick E (2006) Determinants of forced expiratory volume in 1 second (FEV1), forced vital capacity (FVC), and FEV1/FVC in chronic spinal cord injury. Arch Phys Med Rehabil 87: 1327-1333.

6. Lahtinen S, Liisanantti J, Poukkanen M, Laurila P (2017) Goal-directed fluid management in free flap surgery for cancer of the head and neck. Minerva Anestesiol 83: 59-68.

7. Kim M, Woo K, Park B, Kang S (2018) Effects of transfusion on free flap survival: searching for an optimal hemoglobin threshold for transfusion. $J$ Reconstr Microsurg 34: 610-615.

8. Wikner J, Beck-Broichsitter B, Schlesinger S, Schon G, Heiland M, et al. (2015) Thromboelastometry: a contribution to perioperative free-flap management. $J$ Craniomaxillofac Surg 43: 1065-1071.

9. Fang L, Liu J, Yu C, Hanasono M, Zheng G, et al. (2018) Intraoperative use of vasopressors does not increase the risk of free flap compromise and failure in cancer patients. Ann Surg 268: 379-384

10. Elsner U, Henrichs M, Gosheger G, Dieckmann R, Nottrott M, et al. (2016) Forequarter amputation: a safe rescue procedure in a curative and palliative setting in high-grade malignoma of the shoulder girdle. World J Surg Oncol 14: 216

11. Kaddoum R, Burgoyne L, Pereiras L, Germain M, Neel M, et al. (2013) Nerve sheath catheter analgesia for forequarter amputation in paediatric oncology patients. Anaesth Intensive Care 41: 671-677.

12. Bhagia SM, Elek EM, Grimer RJ, Carter SR, Tillman RM (1997) Forequarter amputation for high-grade malignant tumours of the shoulder girdle. J Bone Joint Surg $\operatorname{Br}$ 79: 924-926.

13. Tsui BC, Mohler D, Caruso TJ, Horn JL (2019) Cervical erector spinae plane block catheter using a thoracic approach: an alternative to brachial plexus blockade for forequarter amputation. Can J Anesth 66: 119-120.

14. Parsons CM, Pimiento JM, Cheong D, Marzban SS, Gonzalez RJ, et al. (2012) The role of radical amputations for extremity tumors: a single institution experience and review of the literature. J Surg Oncol 105: 144-155.

15. Clark MA, Thomas JM (2003) Review: Amputation for soft-tissue sarcoma. Lancet Oncol 4: 335-342.

16. Sahu A, Sagar R, Sarkar S, Sagar S (2016) Psychological effects of amputation: A review of studies from India. Ind Psychiatry J 25: 4-10.

Copyright: (C2020 Stoker A. This is an open-access article distributed under the terms of the Creative Commons Attribution License, which permits unrestricted use, distribution, and reproduction in any medium, provided the original author and source are credited. 